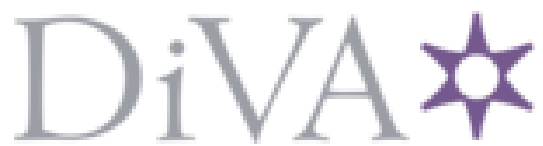

http://www.diva-portal.org

This is the published version of a paper published in Optics Letters.

Citation for the original published paper (version of record):

Zhao, G., Hausmaninger, T., Ma, W., Axner, O. (2018)

Shot-noise-limited Doppler-broadened noise-immune cavity-enhanced optical heterodyne molecular spectrometry

Optics Letters, 43(4): 715-718

https://doi.org/10.1364/OL.43.000715

Access to the published version may require subscription.

N.B. When citing this work, cite the original published paper.

Permanent link to this version:

http://urn.kb.se/resolve?urn=urn:nbn:se:umu:diva-145590 


\title{
Shot-noise-limited Doppler-broadened noise-immune cavity-enhanced optical heterodyne molecular spectrometry
}

\author{
Gang ZhaO, ${ }^{1,2,3}$ (D) Thomas Hausmaninger, ${ }^{2}$ (i) Weiguang Ma, ${ }^{1,3,4}$ (i) and Ove Axner ${ }^{2, *}$ (i) \\ ${ }^{1}$ State Key Laboratory of Quantum Optics \& Quantum Optics Devices, Institute of Laser Spectroscopy, Shanxi University, 030006 Taiyuan, China \\ ${ }^{2}$ Department of Physics, Umeå University, SE-901 87 Umeå, Sweden \\ ${ }^{3}$ Collaborative Innovation Center of Extreme Optics, Shanxi University, 030006 Taiyuan, China \\ ${ }^{4} e$-mail: mwg@sxu.edu.cn \\ *Corresponding author: ove.axner@umu.se
}

Received 30 November 2017; accepted 28 December 2017; posted 10 January 2018 (Doc. ID 314714); published 7 February 2018

\begin{abstract}
Shot-noise-limited Doppler-broadened (Db) noise-immune cavity-enhanced optical heterodyne molecular spectrometry (NICE-OHMS) has been realized by implementation of balanced detection. A characterization of the system based on Allan-Werle plots of the absorption coefficient, retrieved by fitting a model function to data, shows that the system has a white noise equivalent absorption per unit length per square root of bandwidth of $2.3 \times 10^{-13} \mathrm{~cm}^{-1} \mathrm{~Hz}^{-1 / 2}$, solely $44 \%$ above the shot noise limit, and a detection sensitivity of $2.2 \times 10^{-14} \mathrm{~cm}^{-1}$ over $200 \mathrm{~s}$, both being unprecedented for Db NICE-OHMS. The white noise response follows the expected inverse square root dependence on power that is representative of a shot-noise-limited response, which confirms that the system is shot-noiselimited. () 2018 Optical Society of America
\end{abstract}

OCIS codes: (300.6310) Spectroscopy, heterodyne; (120.6200) Spectrometers and spectroscopic instrumentation; (140.4780) Optical resonators.

https://doi.org/10.1364/OL.43.000715

Provided under the terms of the OSA Open Access Publishing Agreement

Noise-immune cavity-enhanced optical heterodyne molecular spectroscopy (NICE-OHMS) is one of the most sensitive techniques for detection of molecular species [1]. It combines cavity-enhanced absorption spectroscopy (CEAS) to increase the interaction length between the light and the analyte, with frequency modulation spectroscopy (FMS), to reduce $1 / f$ noise, especially relative intensity noise. Since the matching of the modulation frequency of the FMS to the free spectral range (FSR) of the cavity provides an immunity to frequencyto-amplitude noise, which is the main limitation in CEAS, it has been prophesied that the technique can be a powerful one for ultrasensitive trace gas detection [2] and that it can provide shot-noise-limited detection under some realistically feasible conditions $[3,4]$.

In fact, by use of a cavity dither paired with wavelength modulation for additional noise reduction, a detection sensitivity of $1 \times 10^{-14} \mathrm{~cm}^{-1}$ over a $1 \mathrm{~s}$ integration time, only 1.5 times above the shot noise limit, was obtained in one of its first realizations [3]. In this case, a well-stabilized fixed-frequency Nd:YAG laser was locked to a cavity with a finesse of $10^{5}$ while addressing a sub-Doppler feature of $\mathrm{C}_{2} \mathrm{HD}$ at $1064 \mathrm{~nm}$. Despite the fact that the technique, since then, has been constructed around a variety of tunable lasers in various configurations [5-17], no system that has provided shot-noise-limited conditions has been realized.

The most persistent development of the technique has been performed based on erbium-doped fiber lasers (EDFL) [7,18-24]. However, despite a series of steadily improved performances, most of which address $\mathrm{Db}$ detection, only detection sensitivities that are significantly above the shot noise limit have been obtained. An exception is the most recent demonstration in which a differential mode of detection was utilized, which could provide a detection sensitivity that was only a factor of 4 above the shot noise limit. The cause for the limited performance of these systems is usually attributed to noise from background signals [8].

Background signals in NICE-OHMS, which are defined as the signals that are measured with an empty cavity, are in general attributed to frequency-dependent dispersion and attenuation in the optical system, which, in turn, are caused by optical components that upset the balance of the triplet. This increases the white noise and brings in long-term drifts in the system [20]. There are two main causes of such signals; one is interference between various optical surfaces, usually termed etalons, while the other is residual amplitude modulation (RAM), which in particular can appear from EOMs [25,26]. Numerous efforts have been made to suppress these types of effects, e.g., antireflection coating, alignment of optical components, active feedback control of EOM [25], usage of proton-exchanged EOMs [20] and wedged crystal EOMs [27], application of etalon immune distance (EID) [28], and implementation of a differential NICEOHMS strategy [24]. However, despite all this, background signals are notoriously difficult to eliminate completely, and the remaining are often the main cause for the restricted performance of the NICE-OHMS system. 
In this Letter, a novel NICE-OHMS system based on balanced detection is presented. The results show that the methodology can strongly reduce background signals generated before the polarizing beam splitter (PBS), which leads to improvements of both the short- and long-term performance of the system. An unprecedented detection sensitivity corresponding to a noise-equivalent absorption per length of $2.2 \times 10^{-14} \mathrm{~cm}^{-1}$ for an integration time of $200 \mathrm{~s}$ is reported. The measured white noise response of the balanced-detection NICE-OHMS, which was found to be $2.3 \times 10^{-13} \mathrm{~cm}^{-1} \mathrm{~Hz}^{-1 / 2}$, is only $44 \%$ above the shot-noise-limited absorption per unit length per square root of bandwidth, $\alpha_{\mathrm{SN}}^{\min }$, which is $1.6 \times 10^{-13} \mathrm{~cm}^{-1} \mathrm{~Hz}^{-1 / 2}$. Moreover, the net white noise response, obtained after subtraction of the detector noise response, adheres to the expected inverse square root dependence of the power that is representative of a shot-noise-limited response. This confirms that the system is shot-noise-limited. This is, to our knowledge, the lowest detection sensitivity and the first time shot-noiselimited detection has been demonstrated for Db NICE-OHMS.

The experimental setup for balanced-detection NICEOHMS, shown in Fig. 1, is similar to that in previous works [22], except for a few minor alterations. In short, the light produced by the EDFL is sequentially passed through a fibercoupled acoustic-optic modulator $(f-\mathrm{AOM})$, a fiber-coupled polarizer $(f$-POL), and a fiber-coupled electro-optic modulator $(f$-EOM) before it is sent out into free space by a fiber collimator $(f-\mathrm{C})$. The $f$-AOM, whose first order diffraction output provides a frequency shift of $110 \mathrm{MHz}$, supplies a highbandwidth $(100 \mathrm{kHz})$ control of the laser frequency for the Pound-Drever-Hall (PDH) locking. Low-frequency feedback (up to $1 \mathrm{kHz}$ ) is fed directly to the piezo in the EDFL. The $f$-EOM, which is equipped with a proton-exchanged waveguide to minimize the generation of RAM, is modulated by two radio frequency signals_one at $20 \mathrm{MHz}$ and another at $380 \mathrm{MHz}$ - to generate the sidebands for the PDH locking and the FMS with modulation indices of 0.1 and 1 , respectively, the latter chosen to maximize the NICE-OHMS signal [29]. Before impinging onto the cavity, the laser propagates through an isolator (ISO), a half-wave plate $(\lambda / 2)$, a modematching lens, a PBS, and a quarter-wave plate $(\lambda / 4)$. To reduce the amount of etalons, the optical components are slightly tilted, parabolic mirrors are used to focus the light onto

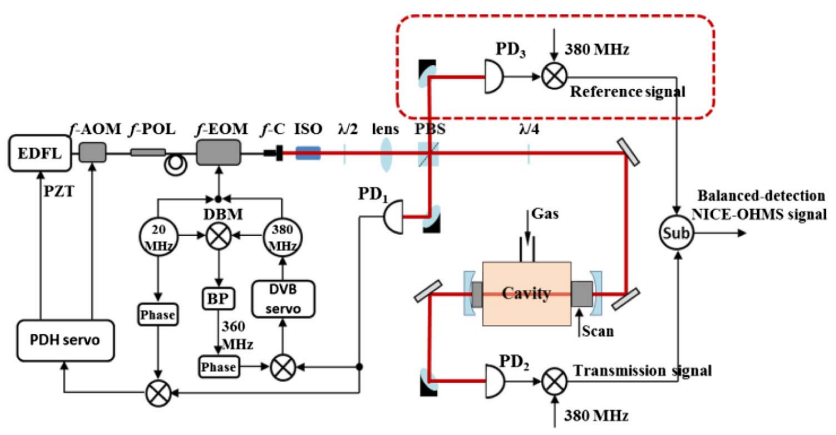

Fig. 1. Experimental setup. EDFL, erbium-doped fiber laser; $f$-AOM, fiber-coupled acousto-optic modulator; $f$-POL, fiber-coupled polarizer; $f$-EOM, fiber-coupled electro-optic modulator; $f$-C, fiber collimator; ISO, isolator; $\lambda / 2$, half-wave plate; PBS, polarizing beam splitter; $\lambda / 4$, quarter-wave plate; $\mathrm{PD}_{1-3}$, photodiodes; $\mathrm{DBM}$, doublebalanced mixer; Sub, subtraction performed in the software. the detectors, and the optical components are, whenever possible, positioned at EID [28].

The cavity is composed of two high-reflectivity mirrors ( $R=99.994 \%$ ), which provide a finesse of 55,000 . They are mounted on PZTs and separated by a Zerodur spacer by a distance of $39.4 \mathrm{~cm}$ to provide a free space range (FSR) of $380 \mathrm{MHz}$. The reflected light of the cavity impinges upon photodetector $\mathrm{PD}_{1}$, whose signal is demodulated to generate the error signals for both the PDH and the DeVoe-Brewer locking [30], where the latter is used to lock the modulation frequency of the FMS to the FSR of the cavity. The transmitted light impinges on photodetector $\mathrm{PD}_{2}$, whose signal is demodulated at the modulation frequency of the FMS $(380 \mathrm{MHz})$ and low-pass filtered with a corner frequency of $1 \mathrm{kHz}$ to produce the NICE-OHMS signal. To scan across the targeted molecular feature \{the $P_{e}(15)$ transition of the $\nu_{1}+\nu_{3}$ band of $\mathrm{C}_{2} \mathrm{H}_{2}$ at $\left.6518.4858 \mathrm{~cm}^{-1}[31,32]\right\}$, a triangle wave (with a frequency of $0.7 \mathrm{~Hz}$ ) is imposed on the front PZT of the cavity. Only the signals during the decreasing section of the triangle wave were sampled, providing a dead time of $0.7 \mathrm{~s}$ between two adjacent samplings.

It had previously been noted that although the EOM has a proton-exchanged waveguide, a temperature-dependent FMS background signal still emerges from the EOM, possibly created by interference between the two surfaces of the crystal in the EOM. The balanced detection realized in this work provides a means to eliminate the influence of any background signal produced in the optical system before the PBS. To realize the balanced detection, a part of the light is deflected by the $\mathrm{PBS}$ onto a third detector $\left(\mathrm{PD}_{3}\right)$, whose signal is demodulated in the same way as the signal from the transmission detector (dashed box) to get a reference signal that contains information about the background signals generated before the PBS, in particular by the EOM. Background signals can then be eliminated by a subtraction of the demodulated NICE-OHMS signal produced by the reference path from the signal measured in transmission, with the former weighted by the ratio of laser powers in the transmission and the reference paths. This provides a balanced detection free of any background signal produced in the optical system before the PBS.

The two panels in Fig. 2 show the drifts of the NICEOHMS background over $4 \mathrm{~h}$, represented by the difference between two empty cavity measurements taken at two instances, separated by $4 \mathrm{~h}$. The red curves in panels (a) and (b) show the background drifts for the transmission and balanced detection, respectively. The black curves represent fits of a

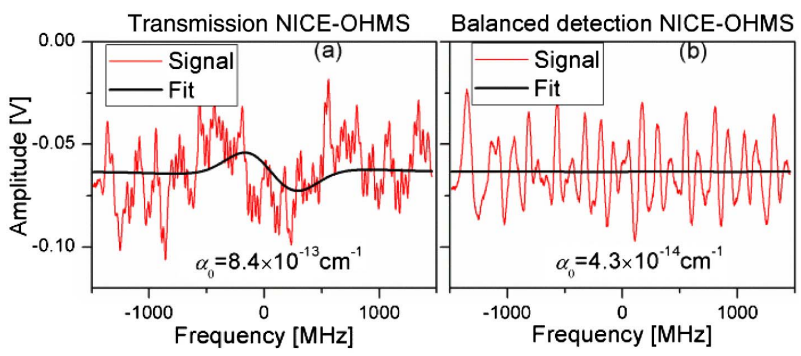

Fig. 2. Red curves: the variation of the NICE-OHMS background signal from an empty cavity by (a) conventional detection (i.e., in transmission) and (b) the balanced detection methodology expressed as the difference between two measurements performed at different time instances, separated by $4 \mathrm{~h}$. Black curves: fits of the Db response. 
Db NICE-OHMS signal to the data based on the model described in [33], and the $\alpha_{0}$ values represent the corresponding amount of drift of the absorption coefficient taken as the amplitude of the fits. The system was calibrated by a standard reference gas with $10 \mathrm{ppm} \mathrm{C}_{2} \mathrm{H}_{2}$ at $50 \mathrm{mTorr}$, according to the process given in [24]. A comparison of the two panels shows that the balanced detection can remove the broad structure from the background signal that is picked up by the fits and will adversely affect the detectability of the $\mathrm{Db}$ response. In this case, the fit to the NICE-OHMS background signal from an empty cavity measured by conventional detection [i.e., in transmission, panel (a)] corresponds to an absorption coefficient of $8.4 \times 10^{-13} \mathrm{~cm}^{-1}$, while the corresponding entity for the case with balanced detection is only $5 \%$ of this, viz. $4.3 \times 10^{-14} \mathrm{~cm}^{-1}$.

Also other types of background signals are decreased by the use of the balanced detection. The narrow periodic structure shown in panel (a), presumed to originate from interference between the output surface of the fiber of the laser and the $f$-C - which are separated by $4 \mathrm{~m}$-is fully eliminated. As can be seen from panel (b), there is though still some remaining structured background. Since the FSR of this is around $120 \mathrm{MHz}$, it is attributed to an etalon created between the cavity front mirror and the transmission detector. However, in comparison with the expected Db NICE-OHMS signal, which can have a $\mathrm{GHz}$ width, such narrow etalon responses will not significantly contribute to fits and, thereby, the assessment of gas. This is the reason the drift of the absorption coefficient retrieved by fitting is significantly reduced (in this case by a factor of 20) when the balanced detection is implemented.

The detection sensitivity of the balanced-detection NICEOHMS was then evaluated by the use of Allan-Werle plots of the absorption coefficient retrieved from fits of a model function of the Db NICE-OHMS to background signals measured at dispersion phase over $12 \mathrm{~h}$ [34]. Figure 3 shows, by the solid curves in black and red, the Allan deviations of the conventional and balanced-detection NICE-OHMS signals from an empty cavity, respectively, as a function of the integration time, $\tau$. The two corresponding dashed lines represent the short-time white noise behavior, which is proportional to $\tau^{-1 / 2}$. The blue dashed line displays the shot-noise-limited response when balanced detection is implemented (see below). The lowermost

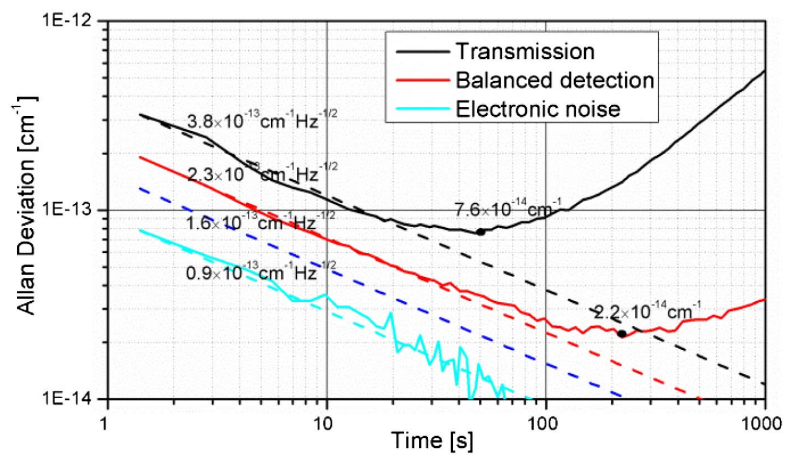

Fig. 3. Two uppermost curves (black and red) display the Allan deviation of the absorption coefficient retrieved by fitting a model function of Db NICE-OHMS to data measured in transmission and by the use of balanced detection, respectively. The corresponding dashed lines show the white noise responses. The solitary dashed line (blue) represents the shot-noise-limited response. The lowermost curve (cyan) shows the detector noise for the balanced mode of detection. curve (cyan) shows the corresponding electronic noise of the balanced system.

For short integration times, the Allan deviations of the NICE-OHMS signals recorded for both modes of detection show a $\tau^{-1 / 2}$-behavior, characteristic of white noise. With increasing measurement time, the effects from other types of noises, e.g., flicker noise and drifts, start to be of importance. For the longest measurement time, the Allan deviations increase with $\tau$, which indicates that drifts start to dominate. For each curve, there is a minimum, whose value should be considered to represent the detection limit of the system (in terms of its absorption coefficient).

The white noise can be compared to $\alpha_{\mathrm{SN}}^{\min }$, which can be measured by turning off the $380 \mathrm{MHz}$ modulation of the laser or using an incoherent light source. Such measurements gave values for $\alpha_{\mathrm{SN}}^{\min }$ of $9.4 \times 10^{-14}$ and $1.5 \times 10^{-13} \mathrm{~cm}^{-1} \mathrm{~Hz}^{-1 / 2}$ for the conventional and balanced mode of detection, respectively.

For comparison, $\alpha_{\mathrm{SN}}^{\mathrm{min}}$ can also be calculated from the detector specifications. The shot noise limit for Db NICE-OHMS by balanced detection, when the assessment of the absorption is made by fitting a model function to a scan, can be expressed as

$$
\alpha_{\mathrm{SN}, \mathrm{bal}}^{\min }=\frac{\pi}{F L} \frac{\sqrt{2} \gamma}{J_{0} J_{1} \kappa} \sqrt{\frac{e \Delta f}{\eta_{A}}} \sqrt{\frac{1+\rho}{\rho}} \sqrt{\frac{1}{P_{t}}},
$$

where $F$ is the finesse of the cavity, $L$ is the cavity length, $\sqrt{2}$ and $\gamma$ take into account the facts that only one half of the scan is used to gather the signal and that the laser is not on resonance all of the time, respectively [22], the $J_{0}$ and $J_{1}$ denote Bessel functions that are functions of the modulation index $\beta, \kappa$ is the ratio of the peak-to-peak value of the NICE-OHMS line shape function and the peak value of the direct absorption line shape, $e$ is the electron charge, $\Delta f$ is the detection bandwidth, $\eta_{A}$ is the detector responsivity, $\rho$ is the ratio of the powers on the reference and the transmission detectors, and $P_{t}$ is the power impinging onto the detector. Inserting relevant numbers for our system, i.e., $L=39.4 \mathrm{~cm} ; \eta_{A}=1 \mathrm{~A} / \mathrm{W} ; \beta=1$, which implies that $J_{0}=$ 0.77 and $J_{1}=0.44 ; \gamma=2.4$ (for a scanning range of $3 \mathrm{GHz}$ ); $\kappa=1.4 ; \quad \Delta f=0.7 \mathrm{~Hz} ; \quad \rho=0.55 ; \quad$ and $P_{t}=2.0 \mathrm{~mW}$, provides a value for $\alpha_{\mathrm{SN}, \mathrm{mal}}^{\min }$ of $1.6 \times 10^{-13} \mathrm{~cm}^{-1} \mathrm{~Hz}^{-1 / 2}$, which compares well with the measured one. A similar agreement can be shown for the conventional mode of detection.

The electronic noise for balanced detection was measuredby blocking the light to the detectors-to be $9.2 \times$ $10^{-14} \mathrm{~cm}^{-1} \mathrm{~Hz}^{-1 / 2}$ (Fig. 3, cyan curve), which is below that of the shot noise.

Figure 3 shows that for conventional detection, i.e., transmitted NICE-OHMS, the white-noise-limited Allan deviation is $3.8 \times 10^{-13} \mathrm{~cm}^{-1} \mathrm{~Hz}^{-1 / 2}$. This is significantly larger than both the shot noise and the electronic noise. The additional amount of white noise, which can be estimated to be $3.6 \times 10^{-13} \mathrm{~cm}^{-1} \mathrm{~Hz}^{-1 / 2}$, is present only if the laser is modulated and can therefore be attributed to processes and background signals associated with the FMS modulation.

Figure 3 shows that when balanced detection was implemented, the white-noise-limited Allan deviation decreased to $2.3 \times 10^{-13} \mathrm{~cm}^{-1} \mathrm{~Hz}^{-1 / 2}$. This indicates that the noise from the FMS modulation process decreased by $90 \%$; the residual contributions (i.e., in addition to shot noise and electronic noise) are attributed to imperfect balanced detection or noncorrelated noise originating after the PBS. The low white 


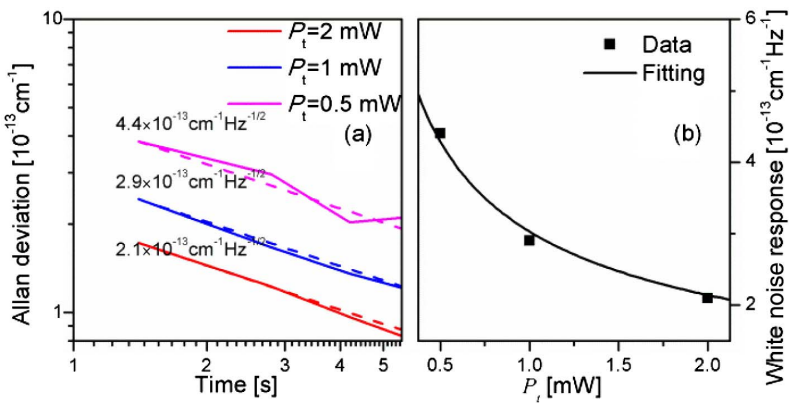

Fig. 4. (a) Allan-Werle plots of balanced-detection NICE-OHMS for three powers. (b) Individual markers: the white noise as a function of transmitted power; solid curve, a fit of the expected shot-noiselimited power dependence. The data represent the net Allan deviations when the contribution from electronic noise has been deducted.

noise level indicates that the balanced mode of detection is shot-noise-limited (it would decrease by $70 \%$ if shot noise would not be present).

For longer measurement times, for which drifts dominate, Fig. 3 shows that for the pertinent experimental system, the balanced mode of detection can improve on the performance of the system considerably. For the longest times displayed in the figure $\left(\sim 10^{3} \mathrm{~s}\right)$, it can reduce the NEAL more than one order of magnitude, from $5.6 \times 10^{-13}$ to $3.4 \times 10^{-14} \mathrm{~cm}^{-1}$. This indicates that the major part of the drifts with the conventional mode of detection originates from the part of the optical system before the PBS.

Figure 3 finally reveals that with the balanced mode of detection, the system can provide a minimum NEAL that is 3 times lower than when conventional detection is used: $2.2 \times 10^{-14} \mathrm{~cm}^{-1}$ at $200 \mathrm{~s}$.

To verify the origin of the white noise response of the system, assessments were performed for three different powers, viz. 100, 50 , and $25 \%$ of the optimum power used above (with a fixed $\rho$ ). Figure 4 shows by panel (a) an Allan-Werle plot of the measurements, while panel (b) displays the white noise response as a function of $P_{t}$. Since the electronic noise is close to the shot noise for the lower laser powers, this was deducted from measured responses, resulting in net white noise responses of $2.1 \times 10^{-13}$, $2.9 \times 10^{-13}$, and $4.4 \times 10^{-13} \mathrm{~cm}^{-1} \mathrm{~Hz}^{-1 / 2}$, respectively. The black curve in panel (b) is a fit of the expected power dependence of shot noise, which, according to Eq. (1), is $P_{t}^{-1 / 2}$. The good agreement confirms that the system is shot-noise-limited.

In summary, a shot-noise-limited Db NICE-OHMS spectrometer has been realized based on balanced detection. It is shown that by subtracting the NICE-OHMS signal from a reference arm from the transmitted signal, background signals generated before the PBS, e.g., from the EOM, the fibers, and other optical components, can be eliminated. The balanced detection can remove a major part of the previously unassigned white noise in NICE-OHMS, resulting in a shot-noise-limited performance with the expected $P_{t}^{-1 / 2}$ power dependence. The balanced detection has also reduced the drifts in the system by more than one order of magnitude, giving rise to an unprecedented detection limit of $2.2 \times 10^{-14} \mathrm{~cm}^{-1}$ at $200 \mathrm{~s}$.

Funding. Vetenskapsrådet (VR) (2015-04374); National Natural Science Foundation of China (NSFC) (61675122).
Acknowledgment. Gang thanks China Scholarship Council (CSC) for support.

\section{REFERENCES}

1. L. S. Ma, J. Ye, P. Dubé, and J. L. Hall, in Laser Spectrosc. XII, M. Inguscio, M. Allegrini, and A. Sasso, eds. (World Scientific, 1995), pp. 199-203.

2. O. Axner, P. Ehlers, A. Foltynowicz, I. Silander, and J. Wang, in Cavity-Enhanced Spectroscopy and Sensing (Springer, 2014), p. 211. 3. J. Ye, L. S. Ma, and J. L. Hall, J. Opt. Soc. Am. B 15, 6 (1998).

4. L. S. Ma, J. Ye, P. Dubé, and J. L. Hall, J. Opt. Soc. Am. B 16, 2255 (1999).

5. J. Bood, A. Mcllroy, and D. L. Osborn, J. Chem. Phys. 124, 084311 (2006).

6. L. Gianfrani, R. W. Fox, and L. Hollberg, J. Opt. Soc. Am. B 16, 2247 (1999).

7. F. M. Schmidt, A. Foltynowicz, W. Ma, and O. Axner, J. Opt. Soc. Am B 24, 1392 (2007).

8. C. L. Bell, G. Hancock, R. Peverall, G. A. D. Ritchie, J. H. van Helden, and N. J. van Leeuwen, Opt. Express 17, 9834 (2009).

9. M. W. Porambo, B. M. Siller, J. M. Pearson, and B. J. McCall, Opt. Lett. 37, 4422 (2012)

10. M. S. Taubman, T. L. Myers, B. D. Cannon, and R. M. Williams, Spectrochim. Acta Part A 60, 3457 (2004).

11. A. Foltynowicz, J. Wang, P. Ehlers, and O. Axner, Opt. Express 18, 18580 (2010)

12. I. Silander, T. Hausmaninger, W. Ma, F. J. M. Harren, and O. Axner, Opt. Lett. 40, 439 (2015).

13. N. J. van Leeuwen and A. C. Wilson, J. Opt. Soc. Am. B 21, 1713 (2004).

14. A. A. Mills, B. M. Siller, M. W. Porambo, M. Perera, H. Kreckel, and B. J. McCall, J. Chem. Phys. 135, 224201 (2011).

15. R. Centeno, J. Mandon, S. M. Cristescu, O. Axner, and F. J. M. Harren, Opt. Express 23, 6277 (2015).

16. T.-L. Chen and Y.-W. Liu, Opt. Lett. 40, 4352 (2015)

17. G. Zhao, T. Hausmaninger, W. Ma, and O. Axner, Opt. Lett. 42, 3109 (2017).

18. F. M. Schmidt, A. Foltynowicz, W. Ma, T. Lock, and O. Axner, Opt. Express 15, 10822 (2007).

19. A. Foltynowicz, W. Ma, and O. Axner, Opt. Express 16, 14689 (2008).

20. A. Foltynowicz, I. Silander, and O. Axner, J. Opt. Soc. Am. B 28, 2797 (2011).

21. P. Ehlers, I. Silander, J. Wang, and O. Axner, J. Opt. Soc. Am. B 29, 1305 (2012)

22. I. Silander, T. Hausmaninger, and O. Axner, J. Opt. Soc. Am. B 32 2104 (2015).

23. I. Silander, T. Hausmaninger, W. Ma, P. Ehlers, and O. Axner, Opt. Lett. 40, 2004 (2015).

24. G. Zhao, T. Hausmaninger, W. G. Ma, and O. Axner, Opt. Express 25, 29454 (2017).

25. W. Zhang, M. J. Martin, C. Benko, J. L. Hall, J. Ye, C. Hagemann, T. Legero, U. Sterr, F. Riehle, G. D. Cole, and M. Aspelmeyer, Opt. Lett. 39, 1980 (2014)

26. I. Silander, P. Ehlers, J. Wang, and O. Axner, J. Opt. Soc. Am. B 29 916 (2012).

27. Z. Li, W. Ma, W. Yang, Y. Wang, and Y. Zheng, Opt. Lett. 41, 3331 (2016).

28. P. Ehlers, A. C. Johansson, I. Silander, A. Foltynowicz, and O. Axner, J. Opt. Soc. Am. B 31, 2938 (2014).

29. P. Ehlers, I. Silander, and O. Axner, J. Opt. Soc. Am. B 31, 2051 (2014).

30. R. G. Devoe and R. G. Brewer, Phys. Rev. A 30, 2827 (1984).

31. "The HITRAN database," http://www.hitran.com.

32. R. El Hachtouki and J. Vander Auwera, J. Mol. Spectrosc. 216, 355 (2002).

33. W. G. Ma, I. Silander, T. Hausmaninger, and O. Axner, J. Quant. Spectrosc. Radiat. Transfer 168, 217 (2016).

34. P. Werle, R. Mucke, and F. Slemr, Appl. Phys. B 57, 131 (1993). 